\title{
DETERMINATION OF PRECESSION AND NUTATION FROM LUNAR LASER RANGING ANALYSIS.
}

\author{
J. G. WILLIAMS, X. X. NEWHALL AND J. O. DICKEY \\ Jet Propulsion Laboratory \\ California Institute of Technology \\ Pasadena CA 91109, USA
}

\begin{abstract}
From the analysis of $24 \mathrm{yr}$ of lunar laser ranges, the correction to the IAU luni-solar precession constant is found to be $-3.2 \pm 0.3 \mathrm{mas} / \mathrm{yr}$ for a total value of $50.3846 " / y r$ at J2000. The size of the $18.6 \mathrm{yr}$ nutation of the pole needs to be increased by about 2 mas over the 1980 IAU theory.
\end{abstract}

\section{Introduction}

The analysis of the Lunar Laser Ranging (LLR) data provides information on a variety of phenomena (Dickey et al. 1994). In this paper the precession rate and $18.6 \mathrm{yr}$ nutation are investigated. Previous LLR solutions for precession and nutation have been given in Williams, Newhall, and Dickey (1991, 1993).

\section{Data}

The lunar laser ranges used in the solutions of this paper consist of 8427 normal points extending from March 1970 to January 1994. The weighted rms residuals during the earliest few years are about $24 \mathrm{~cm}$, and the ranges of recent years have rms residuals of $3 \mathrm{~cm}$. Because of the long time span needed to separate the $18.6 \mathrm{yr}$ nutation from the precession rate, the early data still play an important role in the solutions. 


\section{Solutions}

Corrections to the IAU expressions for precession (Lieske et al. 1977) and nutation (Seidelmann 1982) are needed. In this paper we restrict our solution parameters for precession and nutation to the luni-solar precession rate (at J2000) and the four $18.6 \mathrm{yr}$ coefficients. Imposed on the solution are other corrections to the IAU expressions which are known to be sizable. Corrections to the in-phase annual and semi-monthly terms and the inand out-of-phase $9.3 \mathrm{yr}$ and semi-annual coefficients are adopted from the ZMOA-1990 series (Herring 1991). A -0.24 mas/yr theoretical correction to the obliquity rate (Williams 1994) is imposed.

The early LLR data, though much less accurate than the modern data, extend the span allowing better separation of the precession rate and 18.6 yr nutation coefficients during the solution. But UT1 and polar motion (UT/PM) are much less well known during the early span of data. To test the sensitivity of the solutions to this early UT/PM and to a new solution parameter, a suite of solutions was made.

(1) Solutions were made with two different UT/PM input files provided by R. S. Gross. One used only optical astrometry to generate the early UT/PM; the other used the early LLR Earth rotation results as well as optical astrometry (Gross 1994).

(2) Solutions were made with different spans of the earliest data excluded.

(3) Other solutions were made with decreased weighting of the early data.

(4) With increased data span we now have become sensitive to a small secular change in the lunar orbit eccentricity. A new solution parameter for eccentricity rate was added to our standard set. Our standard tidal model for orbit perturbations has accounted well for tidal changes in the lunar mean distance and orbital period.

Several conclusions were drawn from the suite of solutions. Solutions which rely on optical astrometry alone for the early UT/PM are less satisfactory than those which include LLR information. The solution for the eccentricity rate correction is significant. Failure to solve for it biases the precession rate -0.1 to $-0.2 \mathrm{mas} / \mathrm{yr}$.

Solutions are presented in Table I below. A solution parameter for lunar eccentricity rate is included, and the input UT/PM file includes the LLR Earth rotation. To a reasonable approximation, the corrections $\Delta \epsilon$ and $\sin \epsilon_{0} \Delta \psi$ to a good nutation model are expected to have a particular ratio. For the in-phase components, that ratio depends on the precession rate correction. The table gives a constrained and an unconstrained solution. The constraints are eqs. 3 and 4 of Williams et al. (1991). Constrained and unconstrained solutions are in agreement. The correction to the luni-solar precession is $-3.2 \pm 0.3 \mathrm{mas} / \mathrm{yr}$ for a total value of $50.3846 " / \mathrm{yr}$ at $\mathrm{J} 2000$. 
The in-phase $18.6 \mathrm{yr}$ correction is about 2 mas larger than the 1980 IAU series; and the out-of-phase $18.6 \mathrm{yr}$ correction is small.

TABLE 1. Corrections to the IAU Precession and Nutation Models

\begin{tabular}{lrr}
\hline & Unconstrained & Constrained \\
Precession (mas/yr) & $-3.2 \pm 0.3$ & $-3.2 \pm 0.3$ \\
& & \\
In-phase 18.6 yr coefficients (mas) & $1.8 \pm 1.2$ & $1.7 \pm 0.8$ \\
$\Delta \epsilon$ & $-2.0 \pm 1.3$ & $-1.6 \pm 1.1$ \\
$\sin \epsilon_{0} \Delta \psi$ & & \\
Out-of-phase 18.6 yr coefficients (mas) & $-0.1 \pm 1.2$ & $0.3 \pm 0.9$ \\
$\Delta \epsilon$ & $0.8 \pm 1.0$ & $0.2 \pm 0.7$ \\
$\sin \epsilon_{0} \Delta \psi$ & & \\
\hline
\end{tabular}

\section{Theoretical Developments}

A revision of the theory of precession has been published recently (Williams 1994). It gives some theoretical improvements, updates constants, incorporates additional theoretical improvements by Kinoshita and Souchay (1990), and uses improved ecliptic motion (Laskar 1986, Simon et al. 1994). The most important theoretical correction to the IAU theory is $\mathbf{- 0 . 2 4}$ mas/yr to the obliquity rate. This correction is an observable motion in space, unlike the much larger classical rate which is ecliptic motion. It arises primarily from a tilt in the lunar orbit plane induced by planetary perturbations (giving $-0.254 \mathrm{mas} / \mathrm{yr}$ ), but has small contributions from planetary torques on the Earth $(-0.014 \mathrm{mas} / \mathrm{yr})$, and tidal interactions between the Earth and moon $(+0.024$ mas/yr). This obliquity rate correction is now being seen in the VLBI analyses. There are also small nutation corrections at $18.6 \mathrm{yr}$ due to the planetary tilt effect in the lunar orbit plane: (in mas) $-0.030 \sin \Omega+0.137 \cos \Omega$ to $\Delta \psi$ and $-0.028 \sin \Omega+0.003 \cos \Omega$ to $\Delta \epsilon$.

The second most important theoretical correction is -0.07 mas $/$ decade ${ }^{2}$ in the accumulated precession, due to the Earth's $J_{2}$ rate. This quadratic term will become observable in about a decade.

For a luni-solar precession correction of $-3.2 \mathrm{mas} / \mathrm{yr}$ the paper gives revised precession polynomials and the relative moment of inertia difference $(C-A) / C=0.003273763$ and the normalized polar moment $C / M R^{2}=$ 0.3307007 , where $M$ is the Earth's mass and $R$ its equatorial radius. 


\section{Obliquity and Equinox}

The relative orientation of the planes of the equator and ecliptic, equivalent to the obliquity and dynamical equinox, is determined by LLR. Both of these quantities enter into the theoretical definition of the precession and nutation angles. From the recent lunar and planetary ephemeris DE 245, the obliquity at $\mathrm{J} 2000$ is $23^{\circ} 26^{\prime} 21.409$ ". This is 39 mas less than the IAU value.

Folkner et al. (1994) compare VLBI and dynamical (ephemeris) reference frames. They deduce that at J2000 the IERS celestial origin will be offset from the dynamical equinox by 78 mas in right ascension and the IERS celestial equator (zero declination) will be tilted by 19 mas to the mean equator.

Acknowledgments. We acknowledge and thank the staffs of the CERGA, Haleakala, and University of Texas McDonald observatories, and the LLR associates. The ephemeris DE 245 was a cooperative effort with E. M. Standish. The research described in this paper was carried out at the Jet Propulsion Laboratory of the California Institute of Technology, under a contract with the National Aeronautics and Space Administration.

\section{References}

Dickey, J. O. et al. (1994), Science, Vol. 265, pp. 482-490.

Folkner, W. M., Charlot, P., Finger, M. H., Newhall, X. X., Williams, J. G., Sovers, O. J., and Standish, E. M. (1994), Astron. Astrophys., Vol. 287, pp. 279-289.

Gross, R. S. (1994), to appear in IERS Technical Notes.

Herring, T. A. (1991), in Proceedings of the 127th Colloquium of the International Astronomical Union, Reference Systems, eds. J. A. Hughes, C. A. Smith, and G. H. Kaplan (U. S. Naval Obs., Washington), pp. 157-166.

Kinoshita, H., and Souchay, J. (1990), Celes. Mech. and Dyn. Astron., Vol. 48, pp. $187-265$.

Laskar, J. (1986), Astron. Astrophys., Vol. 157, pp. 59-70.

Lieske, J. H., Lederle, T., Fricke, W., and Morando, B. (1977), Astron. Astrophys., Vol. 58 , pp. 1-16.

Seidelmann, P. K. (1982), Celestial Mech., Vol. 27, pp. 79-106.

Simon, J. L., Bretagnon, P., Chapront, J., Chapront-Touzé, M., Francou, G., and Laskar, J. (1994), Astron. Astrophys., Vol. 282, pp. 663-683.

Williams, J. G. (1994), Astron. J., Vol. 108, pp. 711-724.

Williams, J. G., Newhall, X. X., and Dickey, J. O. (1991), Astron. Astrophys., Vol. 241, pp. L9-L12.

Williams, J. G., Newhall, X. X., and Dickey, J. O. (1993), in Contributions of Space Geodesy to Geodynamics: Earth Dynamics, American Geophysical Union Geodynamics Series, Vol. 24, eds. D. E. Smith and D. L. Turcotte, Washington, D. C., pp. 83-88. 